\title{
Constrictive Pericarditis: Rapid Progression of Pericardial Calcification in a Patient With Hemodialysis and Coronary Artery Bypass Surgery
}

\author{
Naoki Onoda a, d, Syu Yamamoto ${ }^{\mathrm{b}}$, Teruaki Ohomiyac ${ }^{\mathrm{c}}$, Takayuki Honjyo ${ }^{\mathrm{a}}$, \\ Yasuyoshi Iwato ${ }^{\mathrm{a}}$, Takeshi Shichijyo ${ }^{\mathrm{b}}$
}

\begin{abstract}
A 72-year-old man, with a history of coronary artery bypass grafting $(\mathrm{CABG})$ undergone19 years previously and end-stage renal disease (ESRD) on hemodialysis since 1 year before, was admitted for evaluation of dropping arterial pressure down 1 hour after starting hemodialysis. On a computed tomography on chest scan, markedly calcified pericardium on the posterior and diaphragmatic sides of the heart was revealed. Pericardium was mostly not calcified 2 years before admission, pre-initiation of hemodialysis, and the thickness of calcified pericardium was obviously increased after initiation of hemodialysis. Constrictive pericarditis (CP) was diagnosed and subsequent pericardiectomy was underwent, and dropping atrial pressure down during hemodialysis disappeared. The main cause of CP was not cardiac surgery undergone previously (postoperative $\mathrm{CP}$ ). It is probable that secondary hyperparathyroidism in ESRD provoked dystrophic calcification of pericardium, which was injured by past $\mathrm{CABG}$, subsequent rigid and thickness of pericardium, and constrictive physiology became obvious.
\end{abstract}

Keywords: Constrictive pericarditis; End-stage renal disease; Hemodialysis; Secondary hyperparathyroidism; Dystrophic calcification

\section{Introduction}

Cardiac surgery is one of the three most common identifiable causes of constrictive pericarditis (CP) in the modern era [1].

\footnotetext{
Manuscript accepted for publication August 7, 2014

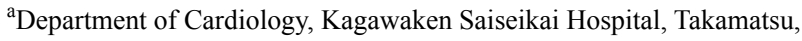
Japan

${ }^{\mathrm{b}}$ Department of Cardiovascular Surgery, Kagawa Prefectural Central Hospital, Takamatsu, Japan

cDepartment of Internal Medicine, Kagawaken Saiseikai Hospital, Takamatsu, Japan

${ }^{\mathrm{d}}$ Corresponding Author: Naoki Onoda, Department of Cardiology, Kagawaken Saiseikai Hospital, 1331-1 Tahikamimachi, Takamatsu, Kagawa 761-8076, Japan. Email: biwafujisakura@yahoo.co.jp

doi: http://dx.doi.org/10.14740/jmc1910w
}

Therefore, it is natural to diagnose postoperative $\mathrm{CP}$ in a patient who underwent past cardiac surgery in daily practice.

We report a case of $\mathrm{CP}$ in a patient who underwent coronary artery bypass grafting (CABG) 19 years previously. We considered the cause of $\mathrm{CP}$ was past cardiac surgery until information of past computed tomography was obtained. Calcification of pericardium was rapidly progressed after initiation of hemodialysis for end-stage renal disease (ESRD), so we consider the cause of $\mathrm{CP}$ is associated with hemodialysis.

\section{Case Report}

A 72-year-old man was admitted for evaluation of dropping arterial pressure down $1 \mathrm{~h}$ after starting hemodialysis. He had a history of hypertension, diabetes mellitus, angina for which he underwent CABG 19 years previously and ESRD on hemodialysis since 1 year before. Since 4 months before admission, paroxysmal atrial fibrillation during hemodialysis emerged and the frequency was gradually increased.

On admission, physical examination revealed no abnormality except for ejection systolic murmur (Levein II/IV) at second left intercostal space at the sternal border. Laboratory findings were as follows: $\mathrm{Hb} 10.0 \mathrm{~g} / \mathrm{dL}$, Hct $31.0 \%$, WBC $7,900 / \mu \mathrm{L}$, platelets $68,000 / \mu \mathrm{L}$, C-reactive protein 0.37 $\mathrm{mg} / \mathrm{dL}$ (normal 0.00 - 0.03), AST $19 \mathrm{IU} / \mathrm{L}$, ALT $22 \mathrm{IU} / \mathrm{L}$, LDH 300 IU/L (normal 106 - 220), ALP 262 IU/L, $\gamma$-GTP 61 IU/L, BUN $57.7 \mathrm{mg} / \mathrm{dL}$, creatinine $4.63 \mathrm{mg} / \mathrm{dL}$, natrium 134 $\mathrm{mEq} / \mathrm{L}$, potassium $4.1 \mathrm{mEq} / \mathrm{L}$, total protein $7.2 \mathrm{~g} / \mathrm{dL}$, albu$\min 3.6 \mathrm{~g} / \mathrm{dL}$, total cholesterol $142 \mathrm{mg} / \mathrm{dL}$, glucose $162 \mathrm{mg} /$ $\mathrm{dL}$, calcium $7.9 \mathrm{mg} / \mathrm{dL}$ (normal 8.2 - 10.8), phosphate 4.3 $\mathrm{mg} / \mathrm{dL}$ (normal 2.6 - 4.5), and parathyroid hormone (PTH) $179 \mathrm{pg} / \mathrm{mL}$ (normal 10 - 65). The other laboratory findings were in normal ranges. Electrocardiography showed normal sinus rhythm with inverted T in I, II, III, aVL, aVf, V3-6 leads (Fig. 1). Chest radiography showed calcification on the posterior and inferior parts of the cardiac silhouette, with four wires fixing the sternum (Fig. 2). On a computed tomography on chest scan, markedly calcified pericardium on the posterior and diaphragmatic sides of the heart was revealed, and it was notable that the thickness of calcified pericardium was increased to approximately twice compared 


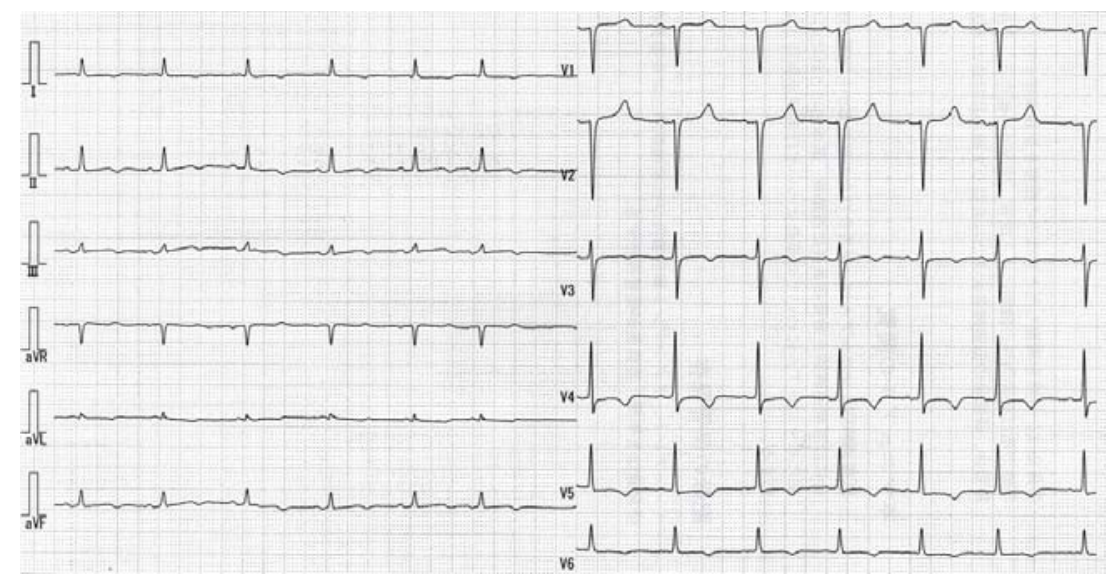

Figure 1. Electrocardiography shows normal sinus rhythm with inverted T in I, II, III, aVL, aVf, V3-6 leads.

to 1 year before admission, 2 months later after initiation of hemodialysis for ESRD. Furthermore, pericardium was mostly not calcified 2 years before admission (Fig. 3).

An echocardiography showed that the size and systolic function of left ventricle were normal, although the left and right atriums were dilated. The transmitral inflow pattern was abnormal with the short deceleration time of early diastole wave. The respiratory early diastolic velocity change of the transmitral inflow was $27 \%$ and of the tricuspid inflow was $43 \%$. Coronary angiogram revealed severe stenosis of the proximal left anterior descending artery and mild stenosis of the mid left circumflex artery, although the left internal thoracic artery to the left anterior descending artery bypass grafting was patent. The hemodynamic measurements showed near equalization of diastolic pressure in all chambers and an early dip and plateau were seen in the pressure tracings of both ventricles (Table 1).

These findings were compatible with chronic CP and

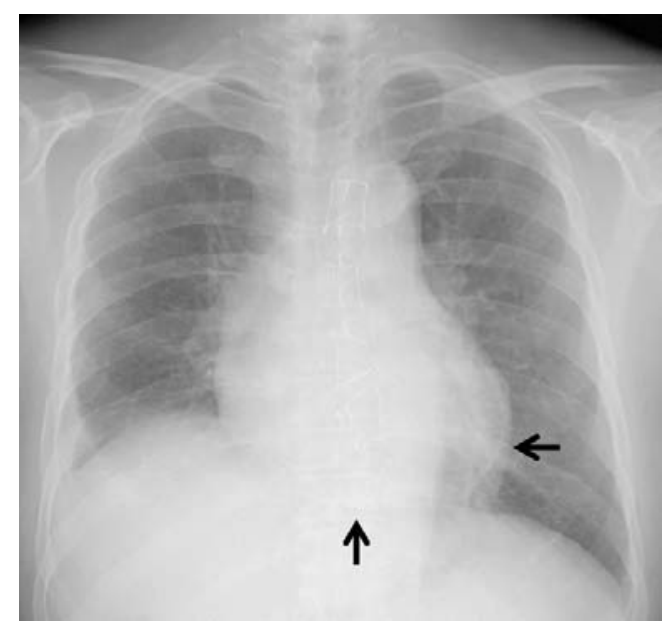

Figure 2. Chest radiography shows calcification on the posterior and inferior parts of the cardiac silhouette (arrow), with four wires fixing the sternum. pericardiectomy was undergone. The pericardium had thickened and tightly adhered to pleura. The diaphragmatic side of pericardium was especially severely calcified and the pericardial space was occupied muddy-necrotizing material which bacterial, acid-fast bacillus cultures or polymerase chain reaction were negative and pathological evaluation was no evidence of caseous necrosis or epithelioid granuloma. Large portions of the adhered pericardium and pleura were resected except for surrounding the LITA-LAD grafting and too rigid posterior part of left atrium.

The postoperative course was uneventful and dropping atrial pressure down during hemodialysis disappeared. Pressure data obtained from cardiac catheterization at 3 weeks after pericardiectomy revealed hemodynamical improvement (Table 1).

\section{Discussion}

The present case was $\mathrm{CP}$ which was successfully treated with partial pericardiectomy. CP is characterized by a thickened, rigid and adherent pericardium which causes restriction of diastolic ventricular filling. The most cases of $\mathrm{CP}$ are thought to follow an episode of acute pericarditis [2,3]. The interesting point of the present case was pericardium was mostly not calcified until initiation of hemodialysis, to wit, calcification of pericardium was confirmed after initiation of hemodialysis, and the thickness of calcified pericardium was obviously increased (approximately twice) in a brief period of 1 year. Hence, here we discuss about the cause of CP of the present case.

In the modern era, the three most common identifiable causes of $\mathrm{CP}$ were cardiac surgery (postoperative $\mathrm{CP}$ ), pericarditis and mediastinal irradiation. Tuberculosis which was once recognized as the most common cause of $\mathrm{CP}$ was declined. Other causes, although less common, were connective tissue disorders, fungal infections, malignancy, trauma, 

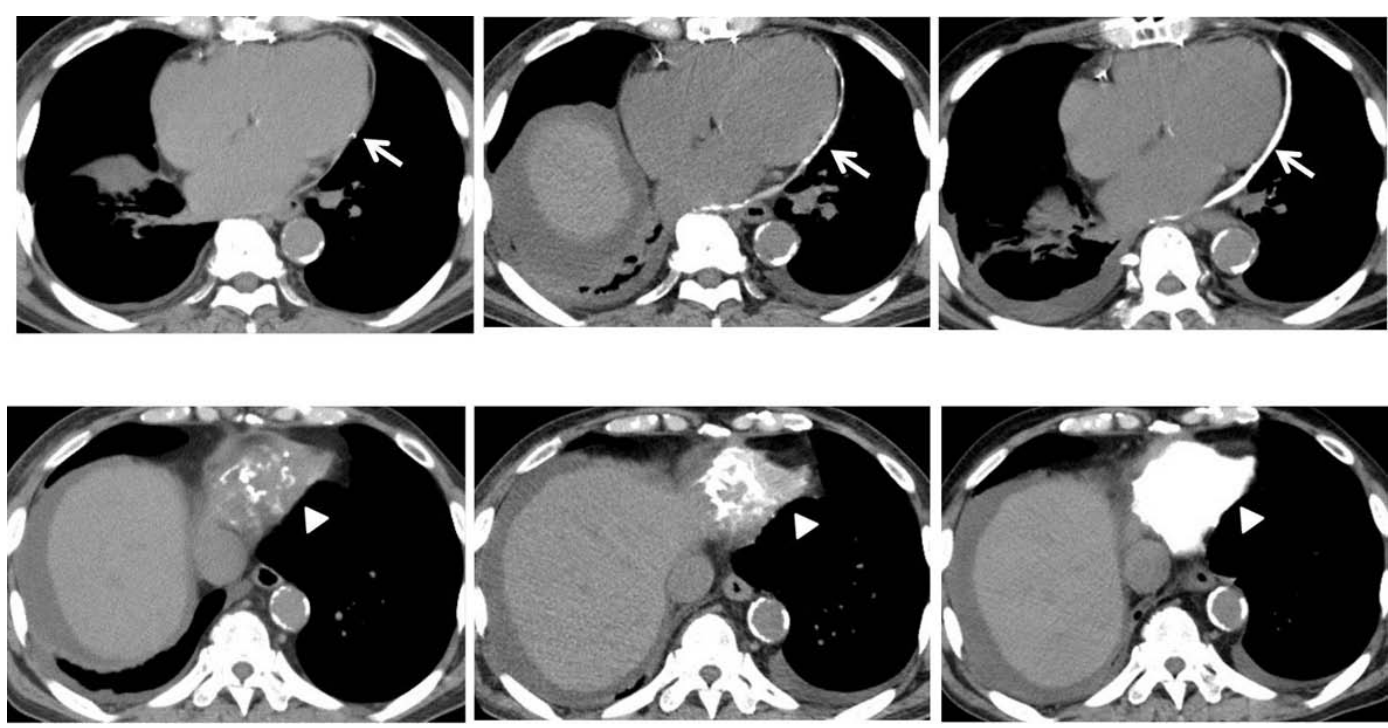

Figure 3. Computed tomography on chest scan (axial view of posterior (upper) and diaphragmatic (lower) sides). Upper row: 2 years before admission (before initiation of hemodialysis) (left). One year before admission (2 months later after initiation of hemodialysis) (middle). At the time of admission (1 year later after initiation of hemodialysis) (right). Lower row: Pericardium was mostly not calcified 2 years before admission (left). Markedly calcified pericardium on the posterior (arrows) and diaphragmatic (arrow-heads) sides of the heart was revealed at the time of admission (right). It was notable the thickness of calcified pericardium was increased to approximately twice compared to 1 year before admission, 2 months later after initiation of hemodialysis (middle).

asbestosis, drug-induced causes and ESRD in hemodialysis $[1,4]$. In the present case, past CABG and ESRD on hemodialysis were probable causes of $\mathrm{CP}$.

Postoperative CP was first reported in 1972 after CABG. The incidence of postoperative $\mathrm{CP}$ is about $2-3 \%$, and the time interval between cardiac surgery and symptoms of $\mathrm{CP}$ varies from 1 to 204 months after cardiac surgery. The causes of postoperative CP are not well known, although several possibilities such as direct trauma to pericardium, pooling of blood, release of proinflammatory cytokines, release of angiogenic growth factors and bacterial contamination induced acute exudative pericarditis, and if not completely resolu-

Table 1. Data of Cardiac Catheterization Pre- and Post-Pericardiectomy

\begin{tabular}{lll}
\hline Pressure (mm Hg) & Pre & Post \\
\hline RA (a/v/m) & $18 / 16 / 15$ & $4 / 2 / 1$ \\
RV (s/d/ed) & $59 / 12 / 20$ & $36 / 0 / 4$ \\
PA (s/d/m) & $56 / 23 / 24$ & $46 / 15 / 26$ \\
PCW (a/v/m) & $36 / 20 / 25$ & $18 / 19 / 15$ \\
LV (s/d/ed) & $147 / 11 / 22$ & Not done \\
Ao (s/d/m) & $144 / 60 / 95$ & Not done \\
\hline
\end{tabular}

RA: right atrium; RV: right ventricle; PA: pulmonary artery; PCW: pulmonary capillary wedge; LV: left ventricle; Ao: aorta; a: a wave; v: v wave; m: mean pressure; s: systolic pressure; d: diastomic pressure; ed: end diastolic pressure. The preoperative hemodynamic measurements showed near equalization of diastolic pressure in all chambers and an early dip and plateau were seen in the pressure tracings of both ventricles. Pressure data obtained from cardiac catheterization at 3 weeks after pericardiectomy revealed hemodynamical improvement. 
tion, ongoing chronic tissue injury following postoperative $\mathrm{CP}$ was developed [5]. In the present case, significant change of pericardium was confirmed after initiation of hemodialysis in the last few years, hence CABG undergone 19 years before was unlikely the main cause of CP.

Pericardial involvement in ESRD is commonly manifested as acute pericarditis (uremic or dialysis pericarditis) and less frequently as chronic CP. Most cases of uremic or dialysis pericarditis was identified of fibrinous or fibrofibrinous pericarditis. It is likely that prolonged adhensive pericarditis with hemorrhage predisposes to subacute constrictive pericarditis, and subsequent fibrosis over a period of years might lead to chronic CP [6]. In the present case, neither symptom (e.g., thoracic pain, cough) or pericardial effusion, which were suspected to be acute pericarditis, had not occurred before or after initiation of hemodialysis. Hence, the possibility of uremic or dialysis pericarditis as a first step of CP was unlikely.

In ESRD, secondary hyperparathyroidism is a common complication, and is characterized by elevated levels of serum PTH. Secondary hyperparathyroidism, hyperphosphatemia, and elevated serum calcium-phosphate product levels lead to extraskeletal calcification and born disease, although their roles remain unexplained [7, 8]. In reported cases of extraskeletal calcification due to secondary hyperparathyroidism in ESRD with renal replacement therapy, the level of serum PTH values was markedly elevated greater than 1,000 $\mathrm{pg} / \mathrm{dL}$, whereas serum phosphate values, calcium-phosphate product were variable: in normal range to elevated range [2, $3,9,10]$. In the present case, however either serum phosphate and calcium-phosphate product was well controlled and serum parathyroid hormone was not markedly elevated; we considered that calcification of pericardium was strongly associated with hemodialysis because it started after initiation of hemodialysis.

Calcification is classified into dystrophic and metastatic calcification. Whereas metastatic calcification occurs in normal tissue with abnormal calcium-phosphate metabolism, dystrophic calcification occurs in abnormal tissue with normal calcium-phosphate metabolism $[10,11]$. We considered secondary hyperparathyroidism promoted dystrophic calcification of pericardium which was injured by past CABG.

In conclusion, the main cause of $\mathrm{CP}$ was neither cardiac surgery undergone previously (postoperative $\mathrm{CP}$ ) nor dialysis pericarditis following CP. It is probable that secondary hyperparathyroidism in ESRD provoked dystrophic calcification of pericardium, which was injured by past $\mathrm{CABG}$, subsequent rigid and thickness of pericardium, and constric- tive physiology became obvious.

\section{References}

1. Marcu CB, Caracciolo E, Donohue T. Rapid progression of pericardial calcification in a patient with end-stage renal disease. Catheter Cardiovasc Interv. 2005;65(1):43-46.

2. Park EA, Lee W, Kim KH, Chung JW, Park JH. Rapid progression of pericardial calcification containing a "calcium paste" in a patient with end-stage renal disease. Circulation. 2011;123(9):e262-264.

3. Ling LH, Oh JK, Schaff HV, Danielson GK, Mahoney DW, Seward JB, Tajik AJ. Constrictive pericarditis in the modern era: evolving clinical spectrum and impact on outcome after pericardiectomy. Circulation. 1999;100(13):1380-1386.

4. Kleynberg RL, Kleynberg VM, Kleynberg LM, Farahmandian D. Chronic constrictive pericarditis in association with end-stage renal disease. Int J Nephrol. 2011;2011:469602.

5. Gaudino M, Anselmi A, Pavone N, Massetti M. Constrictive pericarditis after cardiac surgery. Ann Thorac Surg. 2013;95(2):731-736.

6. Alpert MA, Ravenscraft MD. Pericardial involvement in end-stage renal disease. Am J Med Sci. 2003;325(4):228236.

7. Moe SM, Drueke TB. Management of secondary hyperparathyroidism: the importance and the challenge of controlling parathyroid hormone levels without elevating calcium, phosphorus, and calcium-phosphorus product. Am J Nephrol. 2003;23(6):369-379.

8. Cunningham J, Locatelli F, Rodriguez M. Secondary hyperparathyroidism: pathogenesis, disease progression, and therapeutic options. Clin J Am Soc Nephrol. 2011;6(4):913-921.

9. Timmins S, Hibbert M. Images in clinical medicine. Metastatic pulmonary calcification. N Engl J Med. 2010;363(26):2547.

10. Lee HU, Youn HJ, Shim BJ, Lee SJ, Park MY, Jeong JU, Gu GM, et al. Porcelain heart: rapid progression of cardiac calcification in a patient with hemodialysis. $\mathrm{J}$ Cardiovasc Ultrasound. 2012;20(4):193-196.

11. Matsui M, Okayama S, Takitsume A, Morimoto K, Samejima K, Uemura S, Saito Y. Heart Failure Associated with Metastatic Myocardial Calcification in a Hemodialysis Patient with Progressive Calcification of the Hand. Cardiorenal Med. 2012;2(4):251-255. 